\title{
Polarization effects in heavy $\Lambda$ decays
}

\author{
Thomas Mannel ${ }^{\text {a,b,1 }}$, Winston Roberts ${ }^{\text {a,2 }}$ and Zbigniew Ryzak ${ }^{\text {a,3 }}$ \\ a Lyman Laboratory of Physics, Harvard University, Cambridge, MA 02138, USA \\ b Deutsches Elektronen Synchrotron - DESY, Notkestraße 85, W-2000 Hamburg, FRG
}

Received 8 November 1990; revised manuscript received 23 November 1990

\begin{abstract}
Within the framework of the heavy quark effective theory, we study the process $\Lambda_{c}^{+} \rightarrow \Lambda \ell^{+} v$, with emphasis on the polarization structure in the decay. Assuming factorization of the amplitude, we perform a similar analysis for the decay $\Lambda_{c}^{+} \rightarrow \Lambda \pi^{+}$. The asymmetry parameter, $\alpha$, is predicted to be -1 for this decay. We point out that in a certain limit, the results we obtain are also valid for the corresponding $\Lambda_{b} \rightarrow \Lambda_{c}$ decays.
\end{abstract}

\section{Introduction}

The physics of hadrons containing heavy quarks has recently enjoyed much theoretical interest [1-15]. This has been largely due to the observation that, in the limit of infinitely massive quarks, symmetries above and beyond those usually associated with QCD arise. In the so-called heavy quark effective theory (HQET) that results, these symmetries have been termed heavy flavor symmetry and spin symmetry.

The heavy flavor symmetry is described by the group $\mathrm{SU}\left(N_{\mathrm{h}}\right)$, where $N_{\mathrm{h}}$ is the number of heavy flavors that exist. This symmetry arises because the physics of any situation is unchanged when one infinitely heavy quark is replaced by any of the other $N_{\mathrm{h}}-1$ infinitely heavy quarks. Spin symmetry arises because the spin degrees of freedom of the heavy quark decouple from the light QCD degrees of freedom in the effective theory. Interactions, masses and other hadronic properties are independent of the spin of the heavy quark, at least to leading order in $\Lambda_{\mathrm{QCD}}$ / $m_{\mathrm{Q}}$ and $\alpha_{\mathrm{QCD}}\left(m_{\mathrm{Q}}\right)$, where $m_{\mathrm{Q}}$ is the mass of the heavy quark.

* Work supported by the National Science Foundation under Grant \#PHY-8714654.

1 Supported by a Grant from Deutsche Forschungsgemeinschaft.

2 Supported in part by the Natural Sciences and Engineering Research Council of Canada.

3 Supported by the Department of Energy under Grant \#DEAC02-76ER03064.
Some of the consequences of these symmetries have been investigated in a number of papers. In particular, the symmetries have been used to derive relations between the form factors that describe the weak decays of hadrons. These relations reduce the number of form factors necessary for the description of any single process. For instance, the six, real, generally independent form factors that must be introduced to describe the semileptonic decays of a baryon are reduced to a single one for the decay $\Lambda_{b} \rightarrow$ $\Lambda_{c} \ell v$, if we assume that $b$ and $c$ quarks satisfy the requirements of HQET. For the transition $\Lambda_{c} \rightarrow \Lambda \ell^{+} v$ in which a heavy quark $\mathrm{c}$ is changed into a light quark $\mathrm{s}$, only two form factors are necessary.

The decrease in the number of independent form factors leads to testable experimental predictions. In addition, when we couple this with the hypothesis that the matrix element for non-leptonic decays factorizes (this is explained in section 3 ), we find that the same form factors which describe the semileptonic decays also describe the non-leptonic ones. Obviously, it increases the scope of the predictions that may be made.

In this paper, we explore, in some detail, implications of HQET for the semileptonic decays $\Lambda_{c}^{+} \rightarrow$ $\Lambda \ell^{+} v$. In addition, we study a simple two-body nonleptonic decay, $\Lambda_{c}^{+} \rightarrow \Lambda \pi^{+}$, in which we use the additional assumption of factorization. In section 2 , we set out the formalism of HQET for $\Lambda_{c}$ decays. In section 3, we examine the semileptonic decay of the $\Lambda_{c}$, as well as the process $\Lambda_{c}^{+} \rightarrow \Lambda \pi^{+}$, while we present our conclusions in section 4 . 


\section{Formalism}

As discussed in ref. [6], heavy quark systems may be described by an effective theory in which the heavy quark part of the lagrangian is given by

$\mathscr{L}_{\text {heavy }}=\int \frac{\mathrm{d}^{3} v}{v_{0}}\left(\mathrm{i} \hbar_{v}^{+} \Phi h_{v}^{+}+\mathrm{i} \hbar_{v}^{-} \Phi h_{v}^{-}\right)$.

$\mathscr{D}$ is the covariant derivative, and $h_{v}^{+}\left(h_{v}^{-}\right)$annihilates (creates) heavy quark (antiquark) states with four-momentum

$P^{\mu}=m_{\mathrm{Q}} v^{\mu}+k^{\mu}$,

and $k_{\mu}$ is of the order $A_{\mathrm{QCD}}$.

The dynamics described by $\mathscr{L}_{\text {heavy }}$ differs from QCD by terms that go to zero as $m_{\mathrm{Q}} \rightarrow \infty$, at least logarithmically, as the differences between the effective theory and QCD are due to terms suppressed by powers of $\alpha_{\mathrm{QCD}}\left(m_{\mathrm{Q}}\right)$ or $A_{\mathrm{QCD}} / m_{\mathrm{Q}}$. If we neglect these effects, different velocity sectors do not communicate, leading to the so-called velocity superselection rule. In each velocity sector of the theory described by (1) there is a new symmetry, called spin symmetry, which arises because QCD interactions of a heavy quark become independent of its spin degrees of freedom in the heavy quark limit. This means that for each $v$ there exists a separate SU(2) spin symmetry. Also, the lagrangian, $\mathscr{L}_{\text {heavy }}$ contains no reference to the mass of the heavy quark, which has been formally sent to infinity. Consequently, if we have different species of heavy quarks which obey $m_{\mathrm{Q}} \gg \Lambda_{\mathrm{QCD}}$ the effective theory has an additional heavy flavor symmetry corresponding to exchanges of heavy quarks.

Let us consider a heavy baryon built out of a heavy quark and two light quarks. The mass $M$ of the baryon in the spin symmetry limit tends to the quark mass $M=m_{\mathrm{Q}}\left[1+\mathrm{O}\left(\Lambda_{\mathrm{QCD}} / m_{\mathrm{Q}}\right)\right]$. Such a baryon is moving with an "infinite" momentum $P^{\mu}=M v^{\mu}$ and obviously the only heavy quark states present in the baryon's wave-function come from the $v^{\mu}$ sector of the theory.

The $\Lambda_{c}$, with structure [ $\left.(\mathrm{ud})_{0} \mathrm{c}\right]_{1 / 2}$, is one of the simplest of the heavy baryons. In HQET the nontrivial Lorentz structure of this baryon is due solely to the two polarization states of the heavy quark. This means that the SU(2) of spin symmetry connects different polarization states of the same particle. Consequently, an ordinary Dirac spinor $u_{\Lambda_{c}}(v)$ may be used to describe the representation of $\Lambda_{c}$ under the large symmetry group $\mathrm{G}=$ Lorentz $\oplus S U(2)_{\text {spin }}$.

As discussed in ref. [12], spin symmetry implies that the current matrix element for the decay $\Lambda_{c} \rightarrow \Lambda$ may be written as

$$
\begin{aligned}
H_{\mu} & \equiv\left\langle\Lambda(P)\left|\overline{\mathrm{s}} L_{\mu} \mathrm{c}\right| \Lambda_{\mathrm{c}}(v)\right\rangle \\
& =\bar{u}_{\Lambda}(P)\left[F_{1}(P \cdot v)+\psi F_{2}(P \cdot v)\right] L_{\mu} u_{\Lambda_{\mathrm{c}}}(v),
\end{aligned}
$$

where $L_{\mu}=\gamma_{\mu}\left(1-\gamma_{5}\right)$ is the left handed current. Note that while we discuss mainly the decays to $\Lambda$ 's, the form above is also valid for the matrix elements of the left handed currents between $\Lambda_{c}$ and any other light baryon of spin $\frac{1}{2}$. In particular, one may use the form of eq. (3) to analyze the decay $\Lambda_{c} \rightarrow \Sigma$ as well as the (Cabibbo-suppressed) decays to nucleons in which there is a $c \rightarrow d$ transition. Note that the similarity extends only to the form of the matrix element (3). For example the functional structure of the form factors $F_{1}$ and $F_{2}$ will depend on the light baryon appearing in the final state.

For decays in which the final baryon is also heavy, a decay of the type $\Lambda_{h} \rightarrow \Sigma_{h}$ is forbidden. This is easily seen by noting that since the spins of the heavy quarks decouple, the light quarks are truly spectators in such a decay. This means that the total angular momenta of the light quarks (and accompanying gluons) must be conserved in the decay. In the case of a light (i.e., normal) $\Sigma$, the strange quark's spin is not expected to decouple, so that the spin of the light "spectators" can change during the process. This is discussed more formally in refs. [11-14].

The form of eq. (3) is to be compared with the general form of the current matrix element of the left handed current for weak baryon decays, which is described by six real form factors:

$$
\begin{aligned}
& \left\langle\mathrm{B}^{\prime}, p^{\prime}\left|\overline{\mathrm{q}}^{\prime} L_{\mu} \mathrm{q}\right| \mathrm{B}, p\right\rangle \\
& \quad=\bar{u}\left(p^{\prime}\right)\left\{\left[f_{1}\left(q^{2}\right) \gamma_{\mu}-\mathrm{i} f_{2}\left(q^{2}\right) \sigma_{\mu \nu} q^{\nu}+f_{3}\left(q^{2}\right) q_{\mu}\right]\right. \\
& \left.\quad+\left[g_{1}\left(q^{2}\right) \gamma_{\mu}-\mathrm{i} g_{2}\left(q^{2}\right) \sigma_{\mu \nu} q^{\nu}+g_{3}\left(q^{2}\right) q_{\mu}\right] \gamma_{5}\right\} u(p) .
\end{aligned}
$$

In terms of these form factors, we find

$$
\begin{aligned}
& f_{1}=-g_{1}=F_{1}+\frac{m}{M} F_{2}, \\
& f_{2}=g_{2}=f_{3}=g_{3}=-\frac{1}{M} F_{2} .
\end{aligned}
$$


In the usual notation, this means that HQET predicts that $G_{\mathrm{A}}=-G_{\mathrm{V}}$. In the next section, we examine the significance of (3) for the decays $\Lambda_{c}^{+} \rightarrow \Lambda \ell^{+} v$ and $\Lambda_{\mathrm{c}}^{+} \rightarrow \Lambda \pi^{+}$. In the limit when $F_{2}=0$, all of our results are also valid for the corresponding $\Lambda_{b} \rightarrow \Lambda_{c}$ processes.

\section{Spin structure of $\Lambda_{c}$ decays}

In the analysis that follows, we retain all possible polarization information about the $\Lambda$ and the $\Lambda_{c}$. We do this since the self-analyzing decay of the $\Lambda$ $\left(\Lambda \rightarrow p \pi^{-}\right)$allows a fairly easy determination of its polarization. In addition, our analysis shows that the polarization of the $\Lambda_{c}$ may also be determined from its decay. This of course assumes that the $\Lambda_{c}$ 's are created with some non-zero polarization. While this is unlikely at $\mathrm{e}^{+} \mathrm{e}^{-}$machines with unpolarized beams, $\Lambda_{c}$ 's are expected to be polarized when they are produced at hadron colliders in processes like $\mathrm{pp} \rightarrow \Lambda_{\mathrm{c}}+\mathrm{X}$ or $p \bar{p} \rightarrow \Lambda_{c} \bar{\Lambda}_{c}$. All of this means that in addition to the spectra of leptons (or $\Lambda$ 's) arising from the decay, the various spin correlation quantities will also be of interest.

As discussed in the previous section, the semileptonic decays of the $\Lambda_{c}$ are described by the matrix element of (3). Moreover, if one assumes that other weak decays may be treated in the factorization approximation, then the hadronic current of (3) will be a part of the matrix element of all such decays. This means that the spin structure of the $\Lambda_{c}$ and the light spin- $\frac{1}{2}$ baryon in all decays of the form $\Lambda_{c} \rightarrow B X$ is contained in (3) (B is a light spin- $-\frac{1}{2}$ baryon ). We thus focus some attention on this matrix element.

By the factorization hypothesis we man simply that the matrix element $M$ for the decay $\Lambda_{c} \rightarrow$ BX may be written as

$$
\begin{aligned}
M & \equiv\left\langle\mathrm{B}(p) \mathrm{X}\left|\overline{\mathrm{q}} L_{\mu} \mathrm{c} x^{\mu}\right| \Lambda_{\mathrm{c}}(v)\right\rangle \\
& =\bar{u}_{\mathrm{B}}(p)\left[F_{1}(p \cdot v)+\psi F_{2}(p \cdot v)\right] L_{\mu} u_{\Lambda_{\mathrm{c}}}(v) X^{\mu},
\end{aligned}
$$

where, symbolically,

$X^{\mu}=\left\langle\mathrm{X}\left|x^{\mu}\right| 0\right\rangle$.

We emphasize that the factorization hypothesis is independent of HQET. Decays of the form $\Lambda_{c} \rightarrow B X$ may, in principle, be studied using the factorization hypothesis and the form of the weak current given in eq. (4). What HQET does for us here is to introduce relationships among the form factors, eq. (5), which lead to a great simplification of the final results.

Note also that, given the assumption of factorization, all weak decays of the form $\Lambda_{c} \rightarrow B X$ are not only described by two form factors as in eq. (6), but that these form factors are the same for all such processes, regardless of the form of $X . F_{1}$ and $F_{2}$ will, in general, depend on the light spin- $\frac{1}{2}$ baryon $B$ involved in the process, but not on $\mathrm{X}$. This means that the decays of the form $\Lambda_{c} \rightarrow B X$ are related to each other, and ultimately to the semileptonic decay $\Lambda_{\mathrm{c}} \rightarrow \mathrm{B} \ell^{+} v$. On the other hand, the validity of the factorization hypothesis may depend on X. We expect that the best chance for the factorization to hold corresponds to the case when the invariant mass of $X$ is small. In our opinion states like $\pi$ or $\rho$ are good candidates and in fact we apply our discussion only to $X=\pi$.

For semileptonic decays, where factorization is exact,

$X^{\mu}=\bar{v} \gamma_{\mu}\left(1-\gamma_{5}\right) u$

is the leptonic current. In the case of a decay where $\mathrm{X}=\pi$, and factorization is believed to be approximately correct we have

$X^{\mu}=f_{\pi} P_{\pi}^{\mu}$.

The spin structure present in eq. (3) is easily analyzed by reducing the $4 \times 4$ Dirac algebra to the $2 \times 2$ Pauli algebra. In the notation of ref. [16] the matrix element may then be written as

$M=|D| \chi_{\Lambda}^{\dagger}(s)[1+\sigma \cdot(R+\mathrm{i} I)] \chi_{\Lambda_{\mathrm{c}}}(S)$,

where $s$ is the spin of the light spin- $\frac{1}{2}$ baryon ( $\Lambda$, say) measured in its own rest frame and $S$ is the spin of the $\Lambda_{\mathrm{c}}$ measured in its rest frame. Writing

$X=X_{1}+\mathrm{i} X_{2}$

( $X_{0}$ is real for the cases in which we are interested), we see that

$D=D_{1}+\mathrm{i} D_{2}$,

with

$D_{1}=\left(F_{1}+F_{2}\right)(E+m) X_{0}-\left(F_{1}-F_{2}\right) \boldsymbol{P} \cdot \boldsymbol{X}_{1}$,

$D_{2}=-\left(F_{1}-F_{2}\right) \boldsymbol{P} \cdot \boldsymbol{X}_{2}$.

Then $\boldsymbol{R}$ and $\boldsymbol{I}$ may be written as 
$\boldsymbol{R}=\frac{a_{1} D_{1}+a_{2} D_{2}}{D_{1}^{2}+D_{2}^{2}}$,

$I=\frac{a_{2} D_{1}-a_{1} D_{2}}{D_{1}^{2}+D_{2}^{2}}$

with

$$
\begin{aligned}
\boldsymbol{a}_{1} & =(E+m)\left(F_{1}+F_{2}\right) \boldsymbol{X}_{1} \\
& +\left(F_{1}-F_{2}\right)\left(\boldsymbol{P} \times \boldsymbol{X}_{2}-X_{0} \boldsymbol{P}\right), \\
\boldsymbol{a}_{2} & =(E+m)\left(F_{1}+F_{2}\right) \boldsymbol{X}_{2}-\left(F_{1}-F_{2}\right) \boldsymbol{P} \times \boldsymbol{X}_{1} .
\end{aligned}
$$

$\boldsymbol{P}$ is the three-momentum of the $\Lambda$, which is assumed to be parallel to the $z$-axis.

The forms of the various differential decay rates obtainable from eq. (10) will depend on whether or not the polarizations of the $\Lambda$ and/or $\Lambda_{c}$ are measured. If both $s$ and $S$ are measured, the differential decay rates are proportional to

$\operatorname{Tr}|M|^{2}=D^{\prime}\left(1+\boldsymbol{s} \cdot \boldsymbol{a}+\boldsymbol{S} \cdot \boldsymbol{A}+\boldsymbol{B} \cdot \boldsymbol{s} \times \boldsymbol{S}+s_{i} S_{j} C_{i j}\right)$,

where again for ease of comparison, we are using the same notation as Bjorken [16]. Expanding and evaluating the traces yields the Bjorken results

$D^{\prime}=|D|^{2}\left(1+R^{2}+I^{2}\right)$,

$A=\frac{2(R-R \times I)}{1+R^{2}+I^{2}}$,

$a=\frac{2(R+R \times I)}{1+R^{2}+I^{2}}$,

$B=\frac{2 I}{1+R^{2}+I^{2}}$,

$C_{i j}=\frac{\left(1-R^{2}-I^{2}\right) \delta_{i j}+2\left(R_{i} R_{j}+I_{i} I_{j}\right)}{1+R^{2}+I^{2}}$.

While much of this has already been discussed in ref. [16], the new information available here is in the forms of $\boldsymbol{R}$ and $\boldsymbol{I}$ predicted by HQET. The above equations mean that measurement of the spin variables $\boldsymbol{a}, \boldsymbol{A}, \boldsymbol{B}$, or $C_{i j}$ will serve to test the predictions of HQET.

In eq. (18) above, if the polarization of either the light baryon or that of the $\Lambda_{c}$ is not measured, this amounts to putting $s$ (or $S$ ) $=0$. For example, if $\Lambda_{c}$ 's are produced with no net polarization, $|M|^{2}$ reduces to
$|M|^{2}=D^{\prime}(1+s \cdot a)$.

Some information is also available from the general forms above. When $\boldsymbol{X}$ is real, and there is no recoil of the final baryon $(P=0), I=0$ and $\boldsymbol{R}=\boldsymbol{X} / X_{0}$. Then $B=0$ and $a=A$. If, in addition, $|X| \ll X_{0}$, then $C_{i j} \approx \delta_{i j}$, and there is maximal spin transfer from the $\Lambda_{c}$ to the light baryon. One possible situation where this may arise is discussed below.

\section{1. $\Lambda_{\mathrm{c}}$ semileptonic decays}

We refer to these decays generically as $\Lambda_{c}^{+} \rightarrow$ $\Lambda \ell^{+} v_{\ell}$. These are probably the most interesting of the $\Lambda_{c}$ semileptonic decays, anyway, since the polarization of the $\Lambda$ may be easily measured. For these decays, the vector $X^{\mu}$ is given by eq. (8). Following Bjorken [16], we evaluate

$\boldsymbol{Y}=\frac{\boldsymbol{X}}{X_{0}}=\frac{\hat{\boldsymbol{p}}_{\ell}+\hat{\boldsymbol{p}}_{\mathrm{v}}+\mathrm{i} \hat{\boldsymbol{p}}_{\ell} \times \hat{\boldsymbol{p}}_{\mathrm{v}}}{1+\hat{\boldsymbol{p}}_{\ell} \cdot \hat{\boldsymbol{p}}_{\mathrm{v}}}$,

where $\hat{\boldsymbol{p}}_{v}, \hat{\boldsymbol{p}}_{\ell}$ are unit vectors in the directions of motion of the neutrino and charged lepton, respectively. Of course $\boldsymbol{p}_{v}$ cannot be measured directly, and one needs to know the momenta of all the other particles involved $\left(\Lambda_{c}, \Lambda, \ell^{+}\right)$in order to evaluate $Y$. This can be done only at collider machines where there is $4 \pi$ detection. In electron-positron colliders, if the beams are unpolarized, $\Lambda_{c}$ 's are pair produced with no net polarization, and the decay matrix element is given by eq. (20). Even in this case experiment should be able to provide the values of $\left|D^{\prime}\right|$ and $|a|$ which in principle may allow a calculation of the form factors $F_{1}$ and $F_{2}$.

Note that in general $\boldsymbol{Y}$ is real when the leptons are back-to-back or collinear. For back-to-back leptons, $\boldsymbol{Y} \approx 0, C_{i j} \approx \delta_{i j}$, and there is complete spin transfer from the $\Lambda_{\mathrm{c}}$ to the $\Lambda$. In other words, in this kinematical limit one can directly measure the polarization of the $\Lambda_{c}$ by measuring the polarization of the $\Lambda$. This might be especially useful in determining whether $\Lambda_{c}$ 's are produced polarized in hadron colliders.

3.2. $A_{\mathrm{c}}^{+} \rightarrow \Lambda \pi^{+}$

Let us now turn to the two-body non-leptonic decays of the $\Lambda_{c}$, such as $\Lambda_{c}^{+} \rightarrow \Lambda \pi^{+}$. In what follows we employ the factorization hypothesis which so far lacks 
any good theoretical justification in general (and for which we offer no justification here). Note, however, that recent work by Dugan and Grinstein [17] argues for the validity of this hypothesis in certain kinematic limits. In addition, factorization seems to work phenomenologically, at least for the decays of heavy mesons. Obviously, we expect that the factorization hypothesis has many limitations. In fact, we agree with the proposition that the only place where the factorization could work corresponds to a two body decay where one of the final particles is a light charged meson that can be produced from the virtual W. Fortunately the decay $\Lambda_{c} \rightarrow \Lambda \pi$ is precisely such a decay.

Mindful of this disclaimer we use eqs. (6) and (9) to write

$M_{\Lambda_{\mathrm{c}} \rightarrow \Lambda \pi}=f_{\pi} \bar{u}_{\Lambda}(P)\left(F_{1}+\psi F_{2}\right) \gamma_{\mu}\left(1-\gamma_{5}\right) u_{\Lambda_{\mathrm{c}}}(v) P_{\pi}^{\mu}$.

Substituting $P_{\pi}=m_{\Lambda_{\mathrm{c}}} v-P$, we find

$M_{\Lambda_{\mathrm{c}} \rightarrow \Lambda \pi}=f_{\pi} \bar{u}_{\Lambda}(P)\left(G_{1}+G_{2} \gamma_{5}\right) u_{\Lambda_{\mathrm{c}}}(v)$,

with

$G_{1}=F_{1}\left(m_{\Lambda_{\mathrm{c}}}-m_{\Lambda}\right)+F_{2}\left(m_{\Lambda_{\mathrm{c}}}+m_{\Lambda}-2 E_{\Lambda}\right)$,

$G_{2}=F_{1}\left(m_{\Lambda_{c}}+m_{\Lambda}\right)-F_{2}\left(m_{\Lambda_{c}}-m_{\Lambda}-2 E_{\Lambda}\right)$,

and we have again assumed that the $\Lambda_{c}$ is at rest. We see immediately that, because of momentum conservation, $\boldsymbol{X} \propto \boldsymbol{P}$, and $\boldsymbol{I}=\boldsymbol{B}=\mathbf{0}$. We remind the reader that $F_{1}$ and $F_{2}$ are real.

In terms of the new form factors $G_{1}$ and $G_{2}$, and the usual hyperon decay parameters $\alpha, \beta$ and $\gamma$, the spin variables of eq. (19) are

$a_{x}=a_{y}=A_{x}=A_{y}=0, \quad a_{z}=A_{z}=\alpha$,

$\boldsymbol{B}=\mathbf{0}, \boldsymbol{\beta}=0$;

$C_{x x}=C_{y y}=\gamma, \quad C_{z z}=1$,

$C_{x z}=C_{z x}=C_{x y}=C_{y x}=C_{y z}=C_{z y}=0$.

In terms of $G_{1}$ and $G_{2}$ we find

$\alpha=\frac{-2 G_{1} G_{2} P}{\left(E_{\Lambda}+m_{\Lambda}\right) G_{1}^{2}+\left(E_{\Lambda}-m_{\Lambda}\right) G_{2}^{2}}$,

$\beta=0$,

$\gamma=\frac{\left(E_{\Lambda}+m_{\Lambda}\right) G_{1}^{2}-\left(E_{\Lambda}-m_{\Lambda}\right) G_{2}^{2}}{\left(E_{\Lambda}+m_{\Lambda}\right) G_{1}^{2}+\left(E_{\Lambda}-m_{\Lambda}\right) G_{2}^{2}}$.
In general the quantities $\alpha, \beta$ and $\gamma$ depend on both form factors $F_{1}$ and $F_{2}$. However, in the limit of negligible pion mass, the form factors $G_{1}$ and $G_{2}$ become

$G_{1} \approx \frac{m_{\Lambda_{c}}-m_{\Lambda}}{m_{\Lambda_{c}}}\left(m_{\Lambda_{c}} F_{1}+m_{\Lambda} F_{2}\right)$

$G_{2} \approx \frac{m_{\Lambda_{c}}+m_{\Lambda}}{m_{\Lambda_{c}}}\left(m_{\Lambda_{c}} F_{1}+m_{\Lambda} F_{2}\right)$

Then $\alpha \approx-1$ and $\gamma \approx 0$, independent of the values $F_{1}$ and $F_{2}$. This is a very strong prediction of HQET. It means that one should expect to observe maximal asymmetry in the angular distribution of the $\Lambda$ 's produced in $\Lambda_{c} \rightarrow \Lambda \pi$ (or of the $\Lambda_{c}$ 's produced in $\Lambda_{b} \rightarrow$ $\Lambda_{\mathrm{c}} \pi$ ). Recent experimental results [18] appear to confirm this prediction, since $\alpha$ has been measured to be $-1.0_{-0.0}^{+0.4}$ for the decay $\Lambda_{c}^{+} \rightarrow \Lambda \pi^{+}$. This suggests that both HQET and factorization appear to be working in this decay.

\section{Conclusions}

In this paper we have discussed some decays of heavy $\Lambda_{h}$ particles. Matrix elements corresponding to these decays are severely constrained by HQET. In particular, semileptonic decays $\Lambda_{h} \rightarrow \Lambda_{h^{\prime}}$ are described by a single real form factor, and semileptonic decays $\Lambda_{h} \rightarrow \Lambda$ are described by two real form factors. Non-leptonic decays for which factorization holds are described by the same form factors.

Spin asymmetry and spin correlation measurements can be used to obtain the values of the form factors describing the weak decays. This should be a good test both of HQET and of the factorization hypothesis. Hopefully the relevant data will soon be available.

\section{Acknowledgement}

We thank B. Bruen and B. Wyslouch at MIT for computational advice and facilities. One of us (T.M.) acknowledges the warm hospitality of Lyman Laboratory of Physics at Harvard University, where most of this work was done. 


\section{References}

[1] M. Voloshin and M. Shifman, Sov. J. Nucl. Phys. 45 (1987) 292; 47 (1988) 511.

[2] E. Eichten and B. Hill, Phys. Lett. B 234 (1990) 511.

[3] H. Politzer and M. Wise, Phys. Lett. B 206 (1988) 681; B 208 (1988) 504.

[4] N. Isgur and M. Wise, Phys. Lett. B 232 (1989) 113; B 237 (1990) 527.

[5] B. Grinstein, Nucl. Phys. B 339 (1990) 253.

[6] H. Georgi, Phys. Lett. B 240 (1990) 447.

[7] J. Bjorken, SLAC preprint SLAC-PUB-5278 (1990).

[8] A. Falk, H. Georgi, B. Grinstein and M. Wise, Nucl. Phys. B 343 (1990) 1;

A. Falk and B. Grinstein, Phys. Lett. B 247 (1990) 406.
[9] T. Mannel, W. Roberts and Z. Ryzak, Phys. Lett. B 248 (1990) 389; B 254 (1991) 274.

[10] T. Mannel and Z. Ryzak, Phys. Lett. B 247 (1990) 412.

[11] H. Georgi, Harvard preprint HUTP-90/A046.

[12] T. Mannel, W. Roberts and Z. Ryzak, Harvard preprint HUTP-90/A047, submitted to Nucl. Phys. B.

[13] N. Isgur and $M$. Wise, University of Toronto preprint UTPT90-03, CalTech preprint CALT-68-1626.

[14] D. Politzer, CalTech preprint CALT-68-1651 (1990).

[15] F. Hussain, J.G. Körner and R. Migneron, Phys. Lett. B 248 (1990) 406.

[16] J. Bjorken, Phys. Rev. D 40 (1989) 1513.

[17] M. Dugan and B. Grinstein, Phys. Lett. B 255 (1990) 583.

[18] CLEO Collab., P. Avery et al., Phys. Rev. Lett. 65 (1990) 2842. 